Article

\title{
GmFAD3A, A $\omega$-3 Fatty Acid Desaturase Gene, Enhances Cold Tolerance and Seed Germination Rate under Low Temperature in Rice
}

\author{
Xin Wang ${ }^{1, \dagger}$, Chao Yu ${ }^{1,+}$, Yi Liu ${ }^{1}$, Lu Yang ${ }^{1}$, Yang $\mathrm{Li}^{2}$, Wen Yao ${ }^{2}$, Yicong Cai ${ }^{3}$, Xin Yan ${ }^{1}$, \\ Shaobo Li ${ }^{1}$, Yaohui Cai ${ }^{4}$, Shaoqing $\mathrm{Li}^{5}$ and Xiaojue Peng ${ }^{1, *}$ \\ 1 Key Laboratory of Molecular Biology and Gene Engineering of Jiangxi Province, College of Life Science, \\ Nanchang University, Nanchang 330031, China \\ 2 National Key Laboratory of Wheat and Maize Crop Science, \\ College of Life Sciences, Henan Agricultural University, Zhengzhou 450002, China \\ 3 Key Laboratory of Crop Physiology, Ecology, and Genetic Breeding, Ministry of Education, \\ College of Agronomy, Jiangxi Agricultural University, Nanchang 330045, China \\ 4 Jiangxi Super-Rice Research and Development Center, Nanchang 330200, China \\ 5 State Key Laboratory of Hybrid Rice, College of Life Sciences, Wuhan University, Wuhan 430072, China \\ * Correspondence: xiaojuepeng@ncu.edu.cn; Tel.: +86-791-8396-9537 \\ + These authors contributed equally to this work.
}

Received: 19 June 2019; Accepted: 30 July 2019; Published: 3 August 2019

check for updates

\begin{abstract}
Low temperature is an environmental stress factor that is always been applied in research on improving crop growth, productivity, and quality of crops. Polyunsaturated fatty acids (PUFAs) play an important role in cold tolerance, so its genetic manipulation of the PUFA contents in crops has led to the modification of cold sensitivity. In this study, we over-expressed an $\omega-3$ fatty acid desaturase from Glycine max (GmFAD3A) drove by a maize ubiquitin promoter in rice. Compared to the wild type (ZH11), ectopic expression of GmFAD3A increased the contents of lipids and total PUFAs. Seed germination rates in GmFAD $3 A$ transgenic rice were enhanced under low temperature $\left(15^{\circ} \mathrm{C}\right)$. Moreover, cold tolerance and survival ratio were significantly improved in GmFAD3A transgenic seedlings. Malondialdehyde (MDA) content in GmFAD3A transgenic rice was lower than that in WT under cold stress, while proline content obviously increased. Meanwhile, the activities of superoxide dismutase (SOD), hydroperoxidase (CAT), and peroxidase (POD) increased substantially in GmFAD3A transgenic rice after $4 \mathrm{~h}$ of cold treatment. Taken together, our results suggest that GmFAD3A can enhances cold tolerance and the seed germination rate at a low temperature in rice through the accumulation of proline content, the synergistic increase of the antioxidant enzymes activity, which finally ameliorated the oxidative damage.
\end{abstract}

Keywords: Oryza sativa; $\omega-3$ fatty acid desaturase; GmFAD3A; cold stress; seed germination

\section{Introduction}

Rice (Oryza sativa L.) is an important food crop that feeds more than half of the world's population [1]. Global food security is being challenged by the convergence of multiple factors, including the continuously growing population, reduced arable land, demand for biofuel production, and abiotic stress [2]. Using genetic engineering biotechnology to improve cold-stress resistance of rice is an alternative strategy. This includes using genetic engineering biotechnology to improve the cold resistance of rice.

Low temperature is one of several environmental stress factors that are applied in research aimed at improving the growth, productivity, and quality of crops [3]. To adapt to the stress, plants have 
developed many ways to balance the effects of stress-induced damage, such as increasing the contents of proline, the activity of detoxifying substances or enzymes such as superoxide dismutase (SOD), and peroxidase (POD) [4]. Cell membranes are major sites of freezing injury and cold acclimation. Membrane fluidity is important to sustain the functional activity of membrane proteins and the membranes themselves and is directly affected by temperature [5]. Lipids are an essential component of cell membranes. Plant seeds store lipids as a food reserve for germination and seedling growth. The predominant component of seed lipids, triacylglycerols (TAGs) are not only essential for human nutrition, but also valuable as feedstocks for various industrial products and biofuels [6]. In plants, the level of polyunsaturated fatty acids (PUFAs) are essential for cold acclimation and is essential for the regulation of cholesterol synthesis and transportation for the maintenance of cellular membranes $[7,8]$.

PUFAs are essential for cold stress. Regulating the expression of the PtFAD2 enzymes could potentially alter PUFAs content in membrane lipids [7]. Genetic manipulation of the levels of PUFAs has led to the modification of cold sensitivity in tobacco plants [9]. Changes in freezing tolerance in hybrid poplar was caused by up- or downregulation of PtFAD2 gene expression [7]. PUFAs are the main component of soybean oil [10]. Genes controlling the oleic acid and PUFA contents in soybean seed oil have been characterized, containing the two oleate desaturase genes GmFAD2-1A and GmFAD2-1B and three linoleate desaturase genes GmFAD3A, GmFAD3B, and GmFAD3C [11,12]. Mutation of GmFAD3 resulted in lower linolenic acid content (from 7\% to 10\%) [10,13]. A tobacco FAD3 expressed in rice could increase $\alpha$-linolenic acid (ALA, C18:3) level up to 2.5-fold [14]. The ALA content was increased up to a 13-fold when soybean FAD3 driven by the maize ubiquitin-1 promoter was introduced in rice [15]. However, the function of GmFAD3A to enhance rice to confer cold tolerance is still unclear.

In this study, the GmFAD3A was allogenous expressed in rice. The ectopic expression of GmFAD3A enhances cold stress tolerance in rice, including seed germination rates at low temperature $\left(15^{\circ} \mathrm{C}\right)$ and cold tolerance at the seeding stage. We also evaluated lipid content, the malondialdehyde (MDA), proline content, superoxide dismutase (SOD), and peroxidase (POD) activities to explain its cold stress tolerance.

\section{Results}

\subsection{Structural Characteristics and Cladogram of GmFAD3A}

Sequence analysis indicated that GmFAD3A (Glyma.14G194300) contained eight exons with a 4, 229-bp genomic sequence (Figure 1a). Annotation of the protein sequence on the Pfam website revealed that amino acids 3-67 and 72-333 of the GmFAD3A protein respectively represented domain of unknown function (DUF) and FA_desaturase conserved domains (Figure 1b), implying that GmFAD3A encodes a fatty acid desaturase.

To compare the evolutionary relationship of FAD homologs in rice and soybean, a Neighbor-Joining phylogenetic tree of 14 proteins with high similarity was constructed (Figure 1c). Nine homologs of FADs were identified in rice. Two GmFAD3A homologs of rice (OsFAD8 and OsFAD7) were the closest to GmFAD3A and were categorized into the same clade including GmFAD3A (Figure 1c). When compared with OsFAD8 and OsFAD7, the identity of GmFAD3A amino acid sequences was $72 \%$ in both cases. 
(a) 5 ,

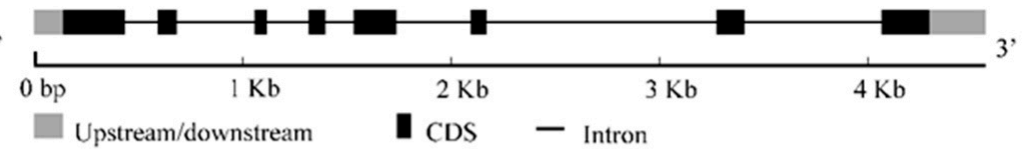

(b)

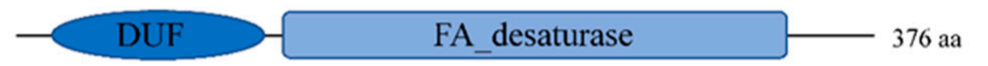

(c)

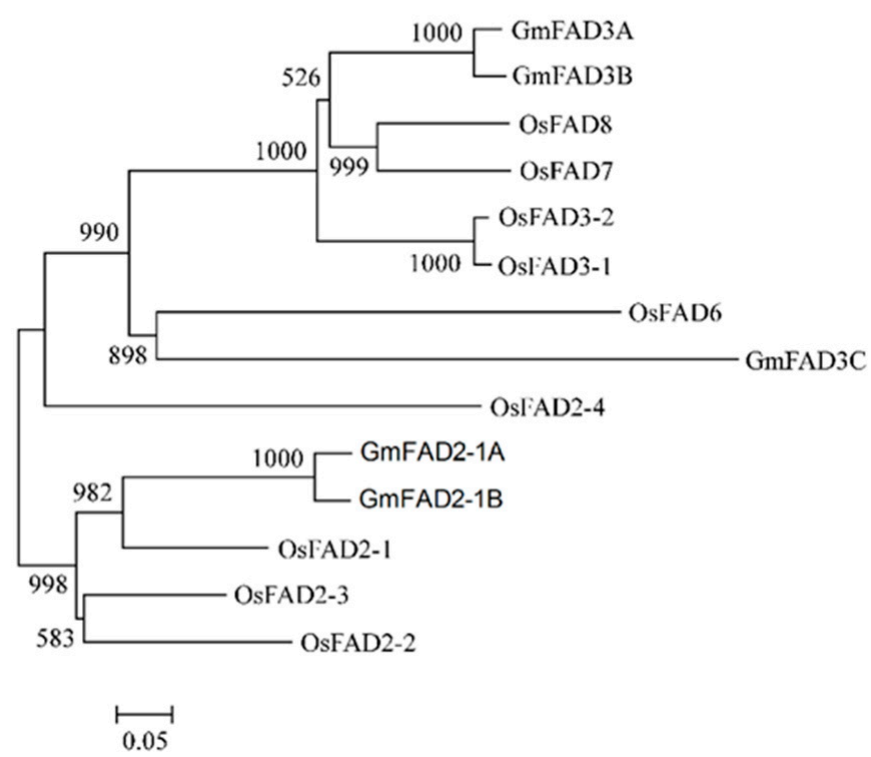

Figure 1. Structural and phylogenetic analyses of GmFAD3A. (a) Schematic representation of the exon (black boxes) and intron (intervening lines) organization of GmFAD3A. (b) A sketch map of protein. The gene encodes a protein of 376 amino acids containing two conserved domains (DUF and FA_desaturase). (c) Phylogenic analysis of GmFADs and OsFADs. There were 14 genes chosen from Oryza sativa and Glycine max.

\subsection{Ectopic Expression of GmFAD3A in Rice}

In order to study the function of GmFAD3A in rice, the gene was cloned from soybean and then transformed into Oryza sativa L. ssp. japonica cv. Zhonghua 11 (ZH11) by constitutive ubiquitin promoter via an Agrobacterium tumefaciens-mediated transformation. More than 40 independent transgenic plants were obtained and most $(>90 \%)$ were positive transgenic plants detected by hygromycin resistance gene (Hpt) PCR analysis (Figure S1a). Four lines (OE4, OE6, OE8, and OE10) with high expression levels compared to ZH11 were selected for going down to the next generation (Figure S1b). Furthermore, two independent transgenic lines (OE4-2 and OE8-5) with high expression levels originating in the $\mathrm{T}_{0}$ generations (OE4 and OE8) were advanced up to the $T_{2}$ generation (Figure 2a,b). All the $T_{2}$ progeny transgenic seeds of OE8-5 lines can grow from hygromycin solution. In addition, every $\mathrm{T}_{2}$ progeny transgenic evens of OE8-5 lines were confirmed by Hpt PCR (Figure S2). 
(a)

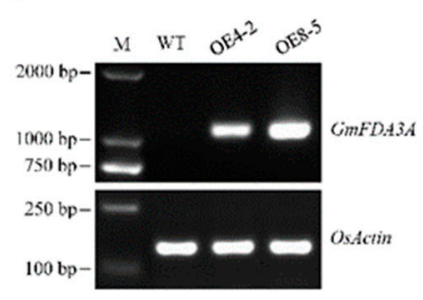

(b)

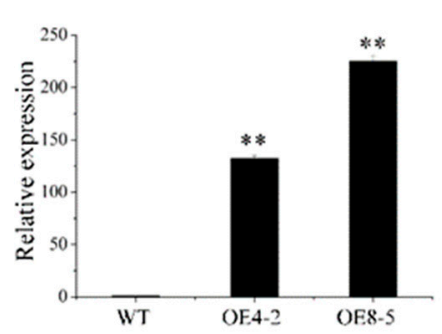

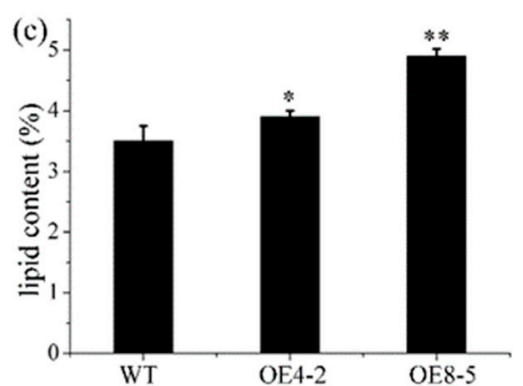

Figure 2. Identification of the GmFAD3A transgenic plants. (a) The GmFAD3A expression in transgenic plants was determined by semi-quantitation RT-PCR analysis. (b) Real-time quantitative PCR analysis in transgenic plants. Values are mean $\pm \operatorname{SD}(n=3)$. (c) Total lipid contents analysis of transgenic rice plants (OE4-2 and OE8-5). Data are mean \pm SE for three replicates. ${ }^{*}, p<0.05 ;{ }^{* *}, p<0.01$.

\subsection{Overexpression of GmFAD3A Increased Lipid Content}

No obvious growth differences were observed between WT and GmFAD3A-OE lines at the vegetative growth stage. The GmFAD3A-OE lines and WT had the same seed setting rate and grain number per panicle (Table S2). Because GmFAD3A is an $\omega-3$ desaturase, lipids of the seeds were examined. The results indicated that the total lipid contents of GmFAD3A-OE lines (OE4-3 and OE8-5) were $11.4 \%$ and $40.4 \%$ higher than that in WT, respectively (Figure 2c). Combined the high expression levels of two independent transgenic lines (OE4-2 and OE8-5) (Figure 2b), there is co-segregation between GmFAD3A overexpression and their phenotypes, indicating that ectopic expression of GmFAD3A increased seed lipid contents.

The crude lipid component of rice includes palmitic acid (C16:0), stearic acid (C18:0), oleic acid (C18:1), linolenic acid (C18:3), arachidic acid (C20:0), and several other components. Palmitic acid is the saturated fatty acid with the highest content in rice seeds, and oleic acid is the predominant monounsaturated fatty acid in rice seed oil [16]. Thus, we further tested the main crude FA composition using GC-MS. The results indicated that the contents of palmitic acid, stearic acid, and arachidic acid did not significantly change, whereas the oleic acid decreased (Table 1). There was a substantial increase in the level of oleic acid $(+5.6 \%$ and $+21.5 \%)$ and the content of linolenic acid $(+25.1 \%$ and $+47.2 \%$ ) in GmFAD3A transgenic lines compared with WT (Table 1). The total polyunsaturated fatty acids levels in OE4-3 (4.271 mg/g) and OE8-5 $(4.983 \mathrm{mg} / \mathrm{g})$ were largely changed compared with WT (3.945 mg/g; Table 1).

Table 1. Fatty acid compositions in Ubi::GmFAD3A $\mathrm{T}_{2}$ transgenic lines and WT (ZH11).

\begin{tabular}{llll}
\hline \multirow{2}{*}{\multicolumn{1}{c}{ Fatty Acid }} & \multicolumn{3}{c}{ Content (mg/g) } \\
\cline { 2 - 4 } & WT & OE4-3 & OE8-5 \\
\hline C13:0 (internal standard) & $1.982 \pm 0.004$ & $1.848 \pm 0.005$ & $1.435 \pm 0.004$ \\
C16:0 (palmitic acid) & $1.414 \pm 0.056$ & $1.587 \pm 0.087$ & $1.566 \pm 0.077$ \\
C18:0 (stearic acid) & $0.263 \pm 0.007$ & $0.273 \pm 0.009$ & $0.275 \pm 0.005$ \\
9cC18:1 (oleic acid) & $3.468 \pm 0.014$ & $3.256 \pm 0.018^{*}$ & $3.186 \pm 0.020^{*}$ \\
C18:2 (linoleic acid) & $3.842 \pm 0.046$ & $4.058 \pm 0.016^{* *}$ & $4.669 \pm 0.014^{* *}$ \\
C18:3n-3 (linolenic acid) & $0.127 \pm 0.013$ & $0.159 \pm 0.015^{*}$ & $0.187 \pm 0.013^{* *}$ \\
C20:0 (arachidic acid) & $0.058 \pm 0.004$ & $0.061 \pm 0.005$ & $0.062 \pm 0.003$ \\
polyunsaturated fatty acids (PUFAs) & $3.945 \pm 0.026$ & $4.271 \pm 0.035^{* *}$ & $4.983 \pm 0.054^{* *}$ \\
\hline
\end{tabular}

\subsection{GmFAD3A Raised Seed Germination Rate at Low Temperature in Rice.}

A previous study showed that stored lipids in seed play a vital role in seed germination and seedling growth [1]. We investigated the seed germination rates of the GmFAD3A-OE line (OE8-5) under $15^{\circ} \mathrm{C}$ and $28^{\circ} \mathrm{C}$. There was no difference in seed germination rate of GmFAD3A-OE lines (OE4-2 and $\mathrm{OE} 8-5)$ at $28{ }^{\circ} \mathrm{C}$, while seed germination rate of GmFAD3A-OE line (OE8-5) (61\%) obviously 
increased under low temperature condition $\left(15^{\circ} \mathrm{C}\right)$ compared to that of WT (about 19.8\%) (Figure 3). These results indicated that ectopic expression of GmFAD $3 A$ enhanced the seed germination rate at low temperature $\left(15^{\circ} \mathrm{C}\right)$ in transgenic rice.
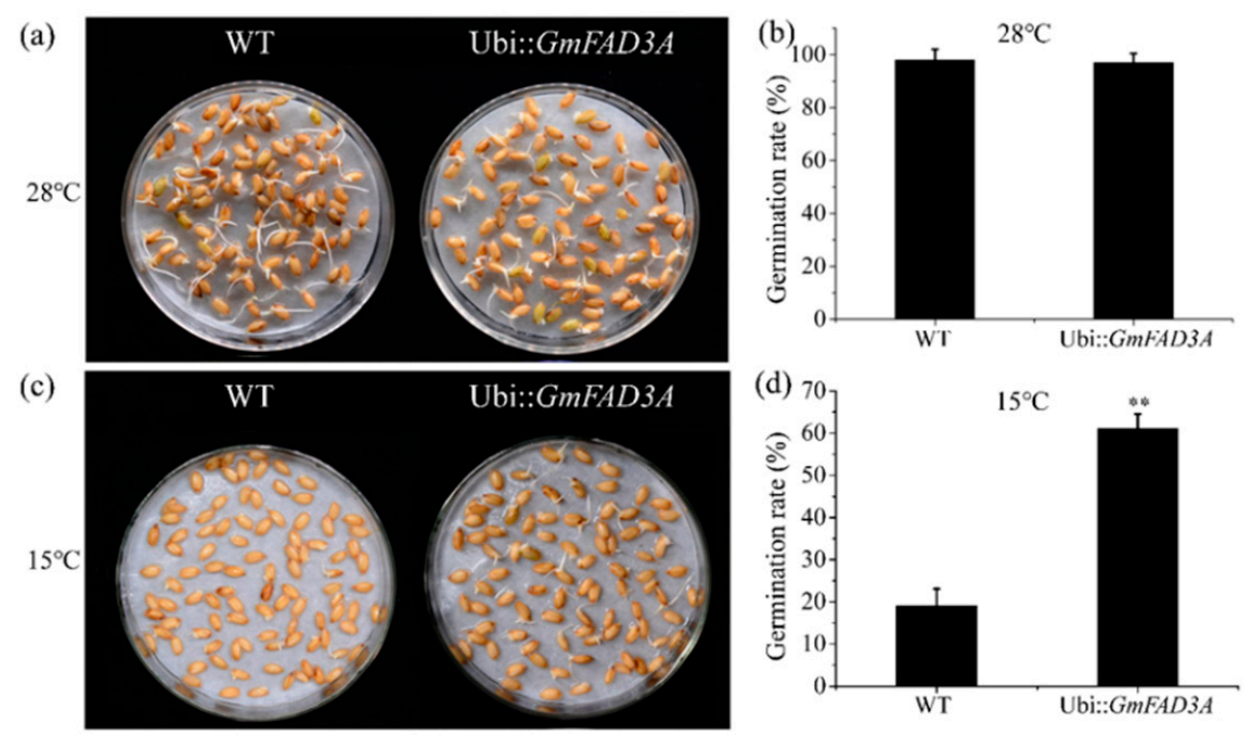

Figure 3. Seed germination assays in mature seeds of wild-type (WT) and GmFAD3A-OE line (OE8-5). $(\mathbf{a}, \mathbf{b})$, seed germination of WT. (c,d), seed germination of GmFAD3A-OE line (OE8-5). Germination was defined as the emergence of the radicles through the seed coat. Data are mean $\pm \mathrm{SE}$ for three replicates with each replicate containing 40 seeds. Data were statistically analyzed vis $t$-test. Asterisks indicate a significant difference between WT and different transgenic lines, ${ }^{* *} p<0.01$.

\subsection{GmFAD3A Enhanced Cold Tolerance in Rice Seedlings}

As the seed germination rate of GmFAD3A-OE lines was affected by low temperature stress (Figure 3), we designed a set of experiments to validate the roles of GmFAD3A at low temperature. 14-d-old rice seedlings grown under normal conditions were transferred into vermiculite for pot experiments under low temperature treatment $\left(4^{\circ} \mathrm{C}\right)$. Following temperature application and recovery, the WT seedlings withered more seriously, compared with GmFAD3A-OE lines (Figure 4). The survival rate of GmFAD3A-OE lines significantly increased. After 7 days of low temperature treatment, approximately $23 \%$ of WT seedings survived; however, the GmFAD3A-OE lines exhibited a $61 \%$ survival rate (Figure $4 \mathrm{~b}$ ). In order to analyze the growth of seedlings, the plant height and growth rate of GmFAD3A-OE lines and WT were measured. The investigation demonstrated that the plant height of WT showed no visible difference, whereas that of GmFAD3A-OE lines increased by $2.03 \mathrm{~cm}$ (Figure 4c). Moreover, the growth rate of the GmFAD3A-OE seedling was $10.17 \%$ which was much higher than that of WT. Taken together, these results confirmed the role of GmFAD3A in cold tolerance. 

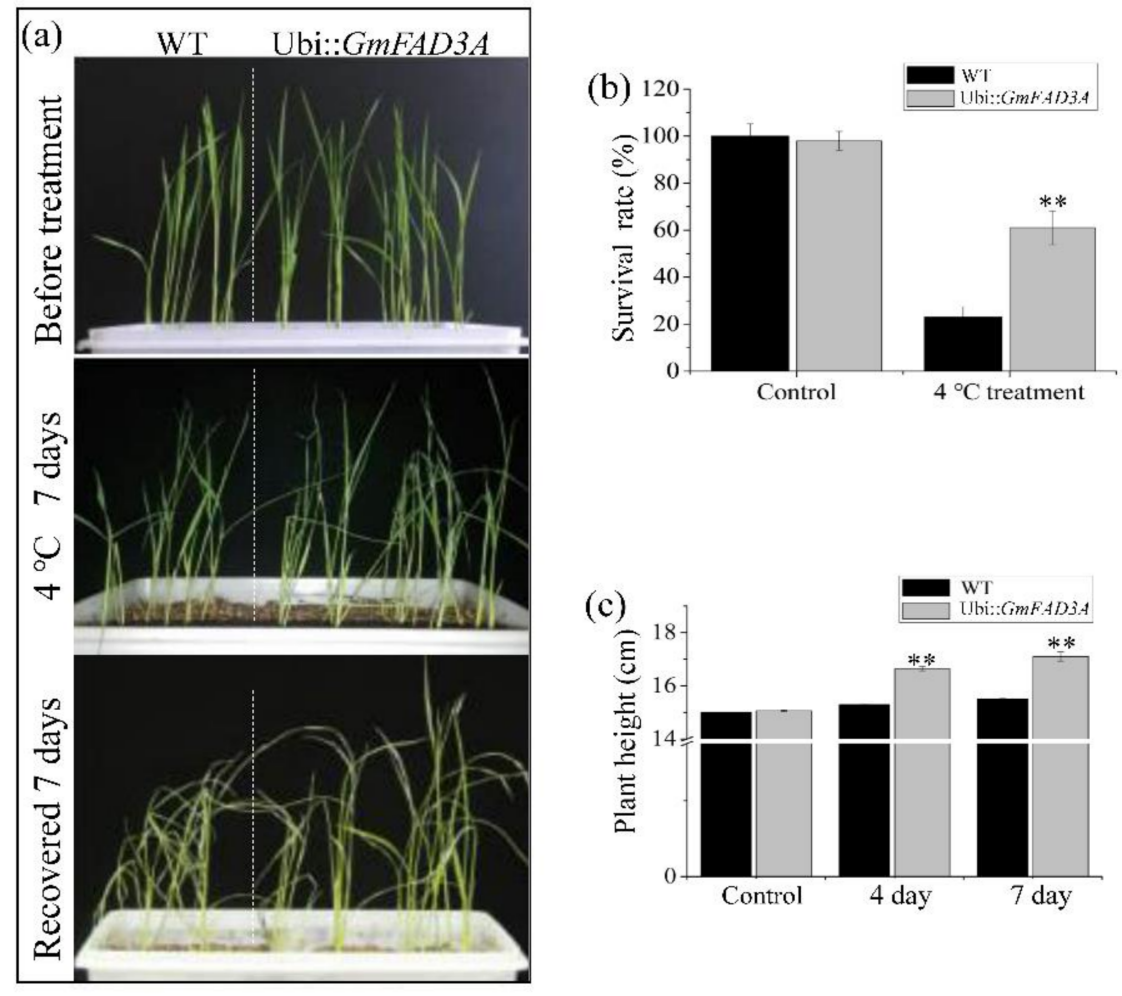

Figure 4. Cold stress tolerance in WT and GmFAD3A-OE plants. 14-d-old seedlings were grown under $4{ }^{\circ} \mathrm{C}$ for 7 days with a 7-day recovery. (a) Representative phenotypes of GmFAD3A-OE line and WT. (b) Survival rate. (c) Plant height. Each biological replicate was the average data collected from 20 plants each of WT and GmFAD3A-OE plants. Bars: SD. ${ }^{* *} p<0.01$.

\subsection{Determination of MDA, Proline Contents, and Antioxidant Enzymatic Activities}

To determine whether ectopic expression of GmFAD3A had an effect on the levels of cold stress-related factors (such as MDA and free proline in rice), we tested these factors in WT and GmFAD3A-OE seedlings under cold stress and under normal conditions. At $0 \mathrm{~h}$, no obvious differences were found in MDA and proline contents between the transgenic plants and WT. Under cold treatment MDA and proline contents in GmFAD3A-OE plants and WT increased. However, MDA content in GmFAD3A-OE plants were significantly lower $(-33.9 \%$ and $-43.8 \%)$ than that of WT after 1 days and 2 days, respectively (Figure 5a). After $1 \mathrm{~h}$ and $2 \mathrm{~h}$ treatment, proline contents in WT and GmFAD3A-OE plants were raised by 2.2, $17.2 \mu \mathrm{g} / \mathrm{mg}$ and 8.7 and $30.4 \mu \mathrm{g} / \mathrm{mg}$, respectively (Figure $5 \mathrm{~b}$ ). Compared to in the WT, the proline content in GmFAD3A-OE plants was obviously increased. The above results showed that ectopic expression of GmFAD $3 A$ in rice could promote the accumulation of free proline, leading to a decline in the content of MDA relative to wild type under cold stress. 

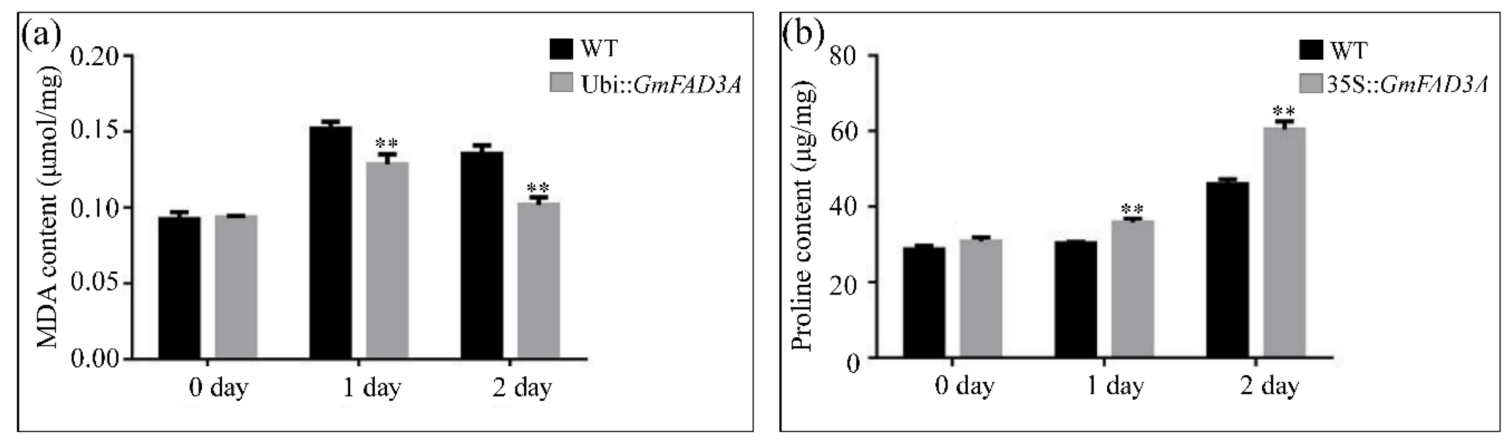

Figure 5. Comparison of MDA and proline content in WT and transgenic line under cold stress. The MDA (a) and proline (b) contents in GmFAD3A-OE and WT plants under cold stress. Data are mean \pm SE for three replicates. Bars: SD. ${ }^{* *} p<0.01$.

We also measured the antioxidant enzymatic activities including superoxide dismutase (SOD), peroxidase (POD), and hydroperoxidase (CAT) under low temperature treatment. The result showed that the activities of these three enzymes in leaf tissues increased under cold treatment (Figure 6). Before cold treatment, the activities of SOD, POD, and CAT in GmFAD3A transgenic plants and WT were no difference. However, under cold conditions the SOD, CAT, and POD activities in GmFAD3A transgenic lines increased significantly compared with those in WT.

(a)

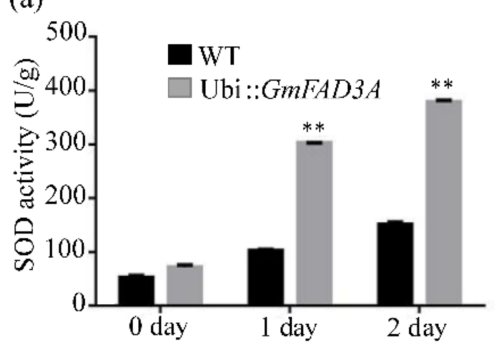

(b)

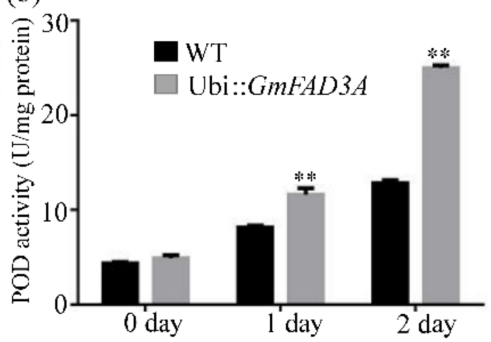

(c)

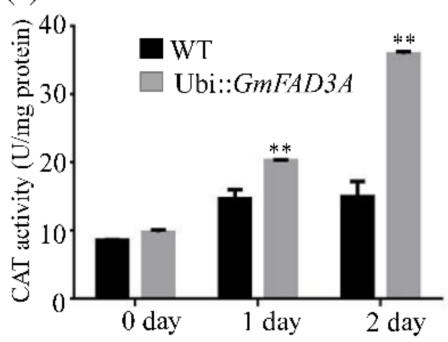

Figure 6. Antioxidant enzyme activity in transgenic and WT plants under cold stress. (a) The SOD levels between WT and transgenic plants. (b) The POD contents between WT and transgenic plants. (c) The CAT activities in WT and transgenic plant. Data are mean \pm SE for three replicates. Bars: SD. ** $p<0.01$.

\section{Discussion}

Abiotic stress is a major concern for agriculture worldwide and is responsible for the loss of crop production [17]. Low temperature is an important damaging factor affecting rice growth, development, and productivity in southern China $[18,19]$. Conventional breeding of cultivated rice has generated increasing yield potential and yield stability. Ectopic expression of the RsICE1 gene enhanced tolerance to low-temperature stress in rice [20]. In this study, we demonstrated that ectopic expression of GmFAD3A increased lipid contents, and resistance to cold stress in rice. Our results provide a new insight into plant-stress tolerance-related genes and will be useful for improving crop resistance.

In plants, the desaturation of linoleic acid (LA) to ALA occurs in plastids and endoplasmic reticulum (ER). Mutants of linoleate desaturase genes (GmFAD3), decreased the linolenic acid content in commercial soybeans by $98 \%$ [10]. Ectopic expression of soybean oleosin genes significantly increased the lipid content in transgenic rice seeds [21]. ER-type $\omega$-3 fatty acid desaturase catalyzes the conversion of 18:2 to 18:3 in phospholipids. The lipid transfer resulting from both sets of $\omega-3$ fatty acid desaturases contributes to the total cellular 18:3 content [22]. The ALA contents in the seeds of GmFAD3A (an ER-localized gene) overexpressing lines increased significantly [23]. In this study, we also detected a substantial increase in level of oleic acid $(+5.6 \%$ and $+21.5 \%)$ and linolenic acid $(+25.1 \%$ and $+47.2 \%)$ 
in GmFAD3A transgenic lines compared with WT. However, whether the GmFAD3A protein has a function in rice growth and cold stress in transgenic rice remains a subject for further investigation.

Polyunsaturated fatty acids (PUFAs) are essential for cold acclimation [7]. Genetic manipulation of the levels of PUFAs has led to the modification of cold tolerance in tobacco and poplar trees $[7,9]$. The unsaturation of lipids has been shown to protect the photosynthetic machinery from photoinhibition at low temperatures [24]. The total polyunsaturated fatty acids levels in GmFAD3A transgenic lines (OE4-3: $4.271 \mathrm{mg} / \mathrm{g}$ and OE8-5: $4.983 \mathrm{mg} / \mathrm{g}$ ) were largely changed compared with WT $(3.945 \mathrm{mg} / \mathrm{g}$; Table 1). The seed germination rates of GmFAD3A transgenic lines increased significantly under low temperature condition $\left(15^{\circ} \mathrm{C}\right)$ compared with WT (Figure 3). Furthermore, the survival and growth rates of GmFAD $3 A$-OE seedlings increased significantly compared with WT at $4{ }^{\circ} \mathrm{C}$. This evidence supported the function of GmFAD3A in cold stress.

Cold stress mediates a series of physiological and metabolite changes, such as alterations in chlorophyll fluorescence, electrolyte leakage, reactive oxygen species (ROS), malondialdehyde (MAD), sucrose, lipid peroxides, proline, and other metabolites [19]. The MDA contents in GmFAD3A transgenic lines were lower (by -0.03 and $-0.04 \mu \mathrm{mol} / \mathrm{mg}$ ) than WT under cold stress after $2 \mathrm{~d}$ and $4 \mathrm{~d}$, respectively. It was suggested that ectopic expression of GmFAD3A in rice reduced the membrane caused lipid peroxidation caused by cold stress. At the same time, the proline content and antioxidant enzymes activity were observed as being higher than that in the WT under cold stress, which might serve as the frontline of defense against oxidative stress. Thus, we suggested that the ectopic expression of GmFAD3A in rice could protect the rice from oxidative damage under cold stress by increasing polyunsaturated fatty acids, antioxidant enzyme activities, and the proline content.

In summary, the data presented here demonstrated that GmFAD3A plays a critical role in cold tolerance and low-temperature germination in rice.

\section{Materials and Methods}

\subsection{Plant Materials and Growth Conditions}

cDNA of soybean (Glycine max) was used as template to amplify the full-length coding sequence of GmFAD3A. Oryza sativa ssp. japonica cv. Zhonghua 11 (ZH11) was used as the background. WT (ZH11) and GmFAD3A transgenic rice were used for the phenotypic trait analyses. WT and transgenic materials were respectively grown under natural field conditions in Nanchang University, Nanchang, China.

\subsection{Structure and Sequence Analysis of GmFAD3A}

The DNA and protein sequences of GmFAD3A were obtained from Phytozome (https://phytozome. jgi.doe.gov/pz/portal.html) and GenBank (http://www.ncbi.nlm.nih.gov/genbank/), respectively. The genomic sequence and protein sequence of GmFAD3A were analyzed using GSDS (http: //gsds.cbi.pku.edu.cn/index.php) and Pfam (http://pfam.sanger.ac.uk/search).

Amino acid sequences of 14 FAD proteins were obtained from rice and soybean. The multiple alignments of amino acid sequences and the neighbor-joining (NJ) phylogenetic tree construction were performed according to previous research [25].

\subsection{Plasmid Construction and Rice Transformation}

The full-length coding region of GmFAD3A was amplified with specific primers FAD3A-C (Table S1) and cloned into a pCAMBIA1301 binary vector. The construction was sequenced to ensure its integrity. The recombinant vector was maintained in Escherichia coli $\mathrm{DH} 5 \alpha$, and then introduced into Agrobacterium tumefaciens EHA105. The construction was transferred into Zhonghua 11 by Agrobacterium-mediated transformation, as previously described [26]. 


\subsection{RNA Isolation and Quantitative RT-PCR Analyses}

The leaves from WT and GmFAD3A transgenic plants used for RNA isolation were frozen in liquid nitrogen and were then stored at $-80^{\circ} \mathrm{C}$. Total RNAs were extracted using TRIzol reagent (TransGen, Beijing, China) according to the manufacturer's protocol. First-strand cDNA was synthesized using PRIME Script Reverse Transcriptase (TaKaRa, Dalian, China, http://www.takara.com.cn/). Quantitative real-time PCR (qRT-PCR) was carried out using an ABI StepOne ${ }^{\mathrm{TM}}$ Real-time PCR instrument (Applied Biosystems, Carlsbad, CA, USA, http://www.appliedbiosystems.com/) and Maxima SYBR Green qPCR Master Mix (Thermo, Waltham, MA, USA). Relative expression levels were calculated via the $2^{-\triangle \Delta C T}$ method [1]. The gene-specific primers used for qRT-PCR are listed in Table S1.

\subsection{Analysis of Lipid Content}

The crude lipid content was studied using the SoxtecTM 2050 Auto Fat Extraction (Foss ${ }^{\circledR}$ Analytic, Hilleroed, Denmark) according to the Soxtec method [1]. Dehulled rice grains were ground to powder. A quantity of $3.3 \mathrm{~g}$ powder was added into the extraction unit, while solvent was added to the extraction cups in a closed system. The extraction consisted of four steps: boiling, rinsing, solvent recovery, and pre-drying. The results were calculated as total amount of fat (g) per $100 \mathrm{~g}$ powder. All measurements were performed with three replicates.

The fatty acid (FA) in seeds was extracted using a chloroform-methanol method according to previous study [27]. Quantification of the FA content was performed using GC-MS referring to the method [28]. C13:0 was used as the internal standard. All measurements were performed with three replicates.

\subsection{Seed Germination Assays}

To determine seed germination, 30 seeds of ZH11 and GmFAD3A $\mathrm{T}_{2}$ homozygous line (OE8-5) were sterilized and spread on 1/2MS medium. Seeds were placed in a growth chamber with light/dark cycle $(12 \mathrm{~h} / 12 \mathrm{~h})$ at $15^{\circ} \mathrm{C}$ or $28^{\circ} \mathrm{C}$. Germination was defined as the emergence of the radicles through the seed coat. The germination rate was calculated from the results of three independent experiments.

\subsection{Cold Stress Tolerance Experiment}

For low-temperature treatment, all rice seedlings including ZH11 and GmFAD3A homozygous line (OE8-5) were grown in 1/2 MS medium and normal conditions $\left(28^{\circ} \mathrm{C}, 16 \mathrm{~h} \mathrm{light}\right.$ and $8 \mathrm{~h}$ dark). 14-day-old seedlings were transferred to a growth chamber at $4{ }^{\circ} \mathrm{C}$ for 7 days. The survival ratio, fresh weight, and plant root or shoot length were calculated after stress or recovery in each pot according to the reported methods [29]. 20 independent transgenic plants and $20 \mathrm{ZH} 11$ seedlings were tested in each replicate. Cold treatments were applied in three independent biological replicates.

\subsection{Physiological and Biochemical Measurements}

Samples for physiological and biochemical measurements assay were collected from non-stressed and cold stressed plants. To measure the free proline (Pro) and malondialdehyde (MDA) contents, $0.2 \mathrm{~g}$ and $0.5 \mathrm{~g}$ of leaves were used for assaying, respectively. All measurements were performed in three biological replicates.

The free proline content was measured by a sulfosalicylic acid method [30]. Quantities of $200 \mathrm{mg}$ (fresh weight) of leaf tissue and $5.0 \mathrm{~mL} \mathrm{3 \%}$ sulfosalicylic acid were added to a mortar for grinding. After centrifugation, $200 \mu \mathrm{L}$ of supernatant was mixed with $400 \mu \mathrm{L}$ of glacial acetic acid and $600 \mu \mathrm{L}$ of $25 \%$ ninhydrin and boiled at $100{ }^{\circ} \mathrm{C}$ for $40 \mathrm{~min}$. After adding toluene, the absorbance was measured at A520.

The MDA level was studied in reference to the thiobarbituric acid-reactive-substances (TBARS) assay in previous reports [31]. Leaf tissues were frozen in liquid nitrogen and ground to powder. A volume of $1.2 \mathrm{~mL}$ of $0.1(w / v)$ trichloroacetic acid (TCA) was added into a tube containing $500 \mathrm{mg}$ of leaf powder, incubated at room temperature for $10 \mathrm{~min}$, then centrifuged at 12,000 rpm for $20 \mathrm{~min}$. An 
aliquot of the supernatant $(0.3 \mathrm{~mL})$ was mixed with $0.3 \mathrm{~mL}$ of $0.5 \%(w / v)$ thiobarbituric acid (TBA), and was incubated at $100{ }^{\circ} \mathrm{C}$ for $20 \mathrm{~min}$. Then, it was quickly cooled, and was centrifuged at $12,000 \mathrm{rpm}$ for 10 min. The A440, A532, and A600 values of the supernatant were recorded.

\subsection{Enzyme Activity Assay}

For the estimation of antioxidant enzyme activities, $1 \mathrm{~g}$ of fresh leaves was ground to powder used liquid nitrogen and then homogenized in $4 \mathrm{~mL}$ of chilled buffer. The homogenate was centrifuged for $15 \mathrm{~min}$ at $12,000 \mathrm{rpm}$ and the supernatant was collected for various enzymatic assays as the described [32]. The enzymatic activity levels of SOD, CAT, and POD were determined using the method given by Wang [30].

\subsection{Statistical Analysis}

All experimental data were the mean of at least three independent replicates, and comparisons between transgenic and WT plants were performed using one-way ANOVA with Duncan's multiple range test. All the statistical analyses were performed using SPSS 12.0 software.

Supplementary Materials: Supplementary materials can be found at http:/www.mdpi.com/1422-0067/20/15/ 3796/s1.

Author Contributions: X.P. and S.L. designed the study. X.W. performed the experiment and wrote the manuscript. C.Y., Y.L. and L.Y. helped in detecting enzymes activity. Y.C. helped in planting the rice. C.Y., S.L. and Y.C. helped in observing the phenotype of plants. W.Y. helped in analyzing the data, Y.L. and X.Y. helped in writing this manuscript. All authors read and approved the final manuscript.

Funding: This research was supported by grants from the National Natural Science Foundation of China (No. 31760377, 31760080, 31401038, and 31360338), the Natural Science Foundation of Jiangxi Province (No. 20181BAB204007), Jiangxi Province Outstanding Young Talents Funding Scheme (No. 20171BCB23021), National Project of Transgenic Crops of Ministry of Science and Technology of China (No. 2016ZX08001-001), and Open Research Fund of State Key Laboratory of Hybrid Rice, Wuhan University (No. KF201601).

Conflicts of Interest: The authors declare no conflict of interest.

\section{Abbreviations}

$\begin{array}{ll}\text { PUFA } & \begin{array}{l}\text { polyunsaturated fatty acid } \\ \text { triacylglycerol }\end{array} \\ \text { TAG } & \text { linoleic acid } \\ \text { LA } & \alpha \text {-linolenic acid } \\ \text { ALA } & \text { malondialdehyde } \\ \text { MDA } & \text { superoxide dismutase } \\ \text { SOD } & \text { peroxidase } \\ \text { POD } & \text { hydroperoxidase } \\ \text { CAT } & \text { reactive oxygen species }\end{array}$

\section{References}

1. Wang, X.; Zhou, W.; Lu, Z.; Ouyang, Y.; Yao, J. A lipid transfer protein, OsLTPL36, is essential for seed development and seed quality in rice. Plant Sci. 2015, 239, 200-208. [CrossRef] [PubMed]

2. Endo, T.; Chiba, B.; Wagatsuma, K.; Saeki, K.; Ando, T.; Shomura, A.; Mizubayashi, T.; Ueda, T.; Yamamoto, T.; Nishio, T. Detection of QTLs for cold tolerance of rice cultivar 'Kuchum' and effect of QTL pyramiding. Theor. Appl. Genet. 2016, 129, 631-640. [CrossRef] [PubMed]

3. Eremina, M.; Rozhon, W.; Poppenberger, B. Hormonal control of cold stress responses in plants. Cell Mol. Life Sci. 2016, 73, 797-810. [CrossRef] [PubMed]

4. Liu, Y.; Xu, C.; Zhu, Y.; Zhang, L.; Chen, T.; Zhou, F.; Chen, H.; Lin, Y. The calcium-dependent kinase OsCPK24 functions in cold stress responses in rice. J. Integr. Plant Biol. 2018, 60, 173-188. [CrossRef] [PubMed] 
5. Beney, L.; Gervais, P. Influence of the fluidity of the membrane on the response of microorganisms to environmental stresses. Appl. Microbiol. Biotechnol. 2001, 57, 34-42. [PubMed]

6. Dyer, J.M.; Mullen, R.T. Engineering plant oils as high-value industrial feedstocks for biorefining: The need for underpinning cell biology research. Physiol. Plant 2008, 132, 11-22. [CrossRef] [PubMed]

7. Zhou, Z.; Wang, M.J.; Zhao, S.T.; Hu, J.J.; Lu, M.Z. Changes in freezing tolerance in hybrid poplar caused by up- and down-regulation of PtFAD2 gene expression. Transgenic Res. 2010, 19, 647-654. [CrossRef] [PubMed]

8. Los, D.A.; Murata, N. Membrane fluidity and its roles in the perception of environmental signals. Biochim Biophys. Acta 2004, 1666, 142-157. [CrossRef] [PubMed]

9. Ishizaki-Nishizawa, O.; Fujii, T.; Azuma, M.; Sekiguchi, K.; Murata, N.; Ohtani, T.; Toguri, T. Low-temperature resistance of higher plants is significantly enhanced by a nonspecific cyanobacterial desaturase. Nat. Biotechnol 1996, 14, 1003-1006. [CrossRef]

10. Pham, A.T.; Shannon, J.G.; Bilyeu, K.D. Combinations of mutant FAD2 and FAD3 genes to produce high oleic acid and low linolenic acid soybean oil. Theor. Appl. Genet. 2012, 125, 503-515. [CrossRef]

11. Schmutz, J.; Cannon, S.B.; Schlueter, J.; Ma, J.; Mitros, T.; Nelson, W.; Hyten, D.L.; Song, Q.; Thelen, J.J.; Cheng, J.; et al. Genome sequence of the palaeopolyploid soybean. Nature 2010, 463, 178-183. [CrossRef]

12. Rajwade, A.V.; Kadoo, N.Y.; Borikar, S.P.; Harsulkar, A.M.; Ghorpade, P.B.; Gupta, V.S. Differential transcriptional activity of SAD, FAD2 and FAD3 desaturase genes in developing seeds of linseed contributes to varietal variation in alpha-linolenic acid content. Phytochemistry 2014, 98, 41-53. [CrossRef] [PubMed]

13. Silva, L.C.C.; Bueno, R.D.; da Matta, L.B.; Pereira, P.H.S.; Mayrink, D.B.; Piovesan, N.D.; Sediyama, C.S.; Fontes, E.P.B.; Cardinal, A.J.; Dal-Bianco, M. Characterization of a new GmFAD3A allele in Brazilian CS303TNKCA soybean cultivar. Theor. Appl. Genet. 2018, 131, 1099-1110. [CrossRef] [PubMed]

14. Shimada, T.; Wakita, Y.; Otani, M.; Iba, K. Modification of Fatty Acid Composition in Rice Plants by Transformation with a Tobacco Microsomal omega;-3 Fatty Acid Desaturase Gene (NtFAD3). Plant Biotechnol. 2000, 17, 43-48. [CrossRef]

15. Anai, T.; Koga, M.; Tanaka, H.; Kinoshita, T.; Rahman, S.M.; Takagi, Y. Improvement of rice (Oryza sativa L.) seed oil quality through introduction of a soybean microsomal omega-3 fatty acid desaturase gene. Plant Cell Rep. 2003, 21, 988-992. [CrossRef] [PubMed]

16. Ying, J.Z.; Shan, J.X.; Gao, J.P.; Zhu, M.Z.; Shi, M.; Lin, H.X. Identification of quantitative trait Loci for lipid metabolism in rice seeds. Mol. Plant 2012, 5, 865-875. [CrossRef] [PubMed]

17. Varshney, R.K.; Bansal, K.C.; Aggarwal, P.K.; Datta, S.K.; Craufurd, P.Q. Agricultural biotechnology for crop improvement in a variable climate: Hope or hype? Trends Plant Sci. 2011, 16, 363-371. [CrossRef]

18. Wang, J.; Wang, J.; Wang, X.; Li, R.; Chen, B. Proteomic response of hybrid wild rice to cold stress at the seedling stage. PLoS ONE 2018, 13, e0198675. [CrossRef]

19. Liu, C.T.; Wang, W.; Mao, B.G.; Chu, C.C. Cold stress tolerance in rice: Physiological changes, molecular mechanism, and future prospects. Yi Chuan 2018, 40, 171-185.

20. Man, L.; Xiang, D.; Wang, L.; Zhang, W.; Wang, X.; Qi, G. Stress-responsive gene RsICE1 from Raphanus sativus increases cold tolerance in rice. Protoplasma 2017, 254, 945-956. [CrossRef]

21. Liu, W.X.; Liu, H.L.; Qu le, Q. Embryo-specific expression of soybean oleosin altered oil body morphogenesis and increased lipid content in transgenic rice seeds. Theor. Appl. Genet. 2013, 126, 2289-2297. [CrossRef]

22. Wang, H.S.; Yu, C.; Tang, X.F.; Wang, L.Y.; Dong, X.C.; Meng, Q.W. Antisense-mediated depletion of tomato endoplasmic reticulum omega-3 fatty acid desaturase enhances thermal tolerance. J. Integr. Plant Biol. 2010, 52, 568-577. [CrossRef] [PubMed]

23. Liu, H.L.; Yin, Z.J.; Xiao, L.; Xu, Y.N.; Qu le, Q. Identification and evaluation of omega-3 fatty acid desaturase genes for hyperfortifying alpha-linolenic acid in transgenic rice seed. J. Exp. Bot. 2012, 63, 3279-3287. [CrossRef]

24. Nishida, I.; Murata, N. CHILLING SENSITIVITY IN PLANTS AND CYANOBACTERIA: The Crucial Contribution of Membrane Lipids. Annu. Rev. Plant Biol. 1996, 47, 541-568. [CrossRef] [PubMed]

25. Cao, Y.P.; Shi, J.L.; Li, Z.; Ming, F. Isolation of OsFAD2, OsFAD6 and FAD family members response to abiotic stresses in Oryza sativa L. Yi chuan= Hereditas 2010, 32, 839-847. [CrossRef] [PubMed]

26. Wu, C.; Li, X.; Yuan, W.; Chen, G.; Kilian, A.; Li, J.; Xu, C.; Li, X.; Zhou, D.X.; Wang, S.; et al. Development of enhancer trap lines for functional analysis of the rice genome. Plant J. 2003, 35, 418-427. [CrossRef] [PubMed]

27. Berry, S. Lipid Analysis: Isolation, Separation, Identification and Structural Analysis of Lipids. Nutr. Bulletin 2010, 29, 72-73. [CrossRef] 
28. Cruz-Hernandez, C.; Deng, Z.; Zhou, J.; Hill, A.R.; Yurawecz, M.P.; Delmonte, P.; Mossoba, M.M.; Dugan, M.E.; Kramer, J.K. Methods for analysis of conjugated linoleic acids and trans-18:1 isomers in dairy fats by using a combination of gas chromatography, silver-ion thin-layer chromatography/gas chromatography, and silver-ion liquid chromatography. J. AOAC Int. 2004, 87, 545-562.

29. Byun, M.Y.; Cui, L.H.; Lee, J.; Park, H.; Lee, A.; Kim, W.T.; Lee, H. Identification of Rice Genes Associated with Enhanced Cold Tolerance by Comparative Transcriptome Analysis with Two Transgenic Rice Plants Overexpressing DaCBF4 or DaCBF7, Isolated From Antarctic Flowering Plant Deschampsia antarctica. Front. Plant Sci. 2018, 9, 601. [CrossRef]

30. Wang, X.; Chen, J.; Liu, C.; Luo, J.; Yan, X.; Aihua, A.; Cai, Y.; Xie, H.; Ding, X.; Peng, X. Over-expression of a protein disulfide isomerase gene from Methanothermobacter thermautotrophicus, enhances heat stress tolerance in rice. Gene 2019, 684, 124-130. [CrossRef]

31. Landi, M. Commentary to: "Improving the thiobarbituric acid-reactive-substances assay for estimating lipid peroxidation in plant tissues containing anthocyanin and other interfering compounds" by Hodges et al., Planta (1999) 207:604-611. Planta 2017, 245, 1067. [CrossRef] [PubMed]

32. Kaur, N.; Dhawan, M.; Sharma, I.; Pati, P.K. Interdependency of Reactive Oxygen Species generating and scavenging system in salt sensitive and salt tolerant cultivars of rice. BMC Plant Biol. 2016, 16, 131. [CrossRef] [PubMed]

(C) 2019 by the authors. Licensee MDPI, Basel, Switzerland. This article is an open access article distributed under the terms and conditions of the Creative Commons Attribution (CC BY) license (http://creativecommons.org/licenses/by/4.0/). 